ИСПОЛЬЗОВАНИЕ ЭКОНОМЕТРИЧЕСКИХ МЕТОДОВ В АНАЛИЗЕ РЫНКА СОВРЕМЕННОЙ ПРОМЫШЛЕННОЙ ИГРУШКИ

\author{
Р. В. Гавва \\ Сергиево-Посадский институт игрушки - филиал \\ Высшей школы народных искусств (академии), Сергиев Посад, Россия
}

Е. Н. Ложкомоева

Сергиево-Посадский филиал Московского педагогического государственного университета, Сергиев Посад, Россия

В статье кратко охарактеризованы основные исторические этапы развития производства промышленной игрушки, рассмотрены вопросы применения эконометрических методов в изучении показателей развития промышленной игрушки. Показано, что специфика отрасли, особенности ее развития отражаются на целесообразности применения конкретных методов, моделей, информационных технологий, способов обработки статистической информации, анализа и прогнозирования ее индикаторов. Отмечаются трудности российских предприятий, производящих игрушки. Определяется необходимость оперативно и грамотно оценивать сложившуюся ситуацию для корректировки деятельности предприятий с целью формирования устойчивых позиций на рынке в будущем. Обозначено, что решение задач развития и проблем отрасли невозможно без проведения глубокого, научно обоснованного анализа состояния рынка промышленной игрушки и оценки перспектив его развития. Особое внимание уделено исследованию возможности использования макроэкономических моделей для анализа развития современной промышленной игрушки. Для того чтобы руководители предприятий могли менять сложившуюся на предприятии ситуацию в сторону улучшения, следует ориентироваться на потребности потенциального потребителя, анализ которых целесообразно проводить на основе применения эконометрических моделей. Рассмотрены конкретные примеры использования эконометрических моделей в анализе показателей рынка промышленной игрушки, сформулированы выводы и даны рекомендации по их прикладному применению.

Ключевые слова: коэффициент корреляции, модель, мультиколлинеарность, современная промышленная игрушка, факторный признак, эконометрическая модель.

\title{
THE USE OF ECONOMETRIC METHODS FOR ANALYZING THE MARKET OF PRESENT DAY MANUFACTURED TOYS
}

\author{
Ruslan V. Gavva \\ Sergiev Posad Institute of Toys - branch of Higher School of Folk Arts (Academy) \\ Sergiev Posad, Russia \\ Evgenia N. Lozhkomoeva \\ Sergiev Posad branch of the Moscow Pedagogical State University, \\ Sergiev Posad, Russia
}

\begin{abstract}
The article provides brief characteristic of key historic stages in the development of manufactured toys and studies issues of applying econometric methods for investigating indicators of manufactured toys development. It is shown that specificity of industry, features of its development influence the expediency of using concrete methods, models, information technologies, ways of processing statistic information, analysis and forecast of its indices. The article points to difficulties of Russian enterprises manufacturing toys. It underlines the necessity to estimate correctly and timely the existing situation in order to correct the work of the enterprise to form stable positions on the market in the future. It is pointed out that resolving problems of development and industry as a whole is impossible without a
\end{abstract}


thorough, scientifically grounded analysis of the market of manufactured toys and forecast of its prospects. Special attention is paid to research of possible use of macro-economic models in order to analyze the development of present day manufactured toys. To give management an opportunity to change the existing situation at the enterprise for the better it is necessary to orient to needs of potential customer, whose analysis can be made on the basis of econometric models. Concrete examples of using econometric models in the analysis of indices of manufactured toys market are provided and recommendations for their applied use are given.

Keywords: correlation coefficient, model, multi-collinear feature, present day manufactured toys, factor sign, econometric model.

\section{Введение}

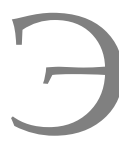
конометрические методы и модели широко применяются в анализе различных сфер и видов экономической деятельности. Наиболее часто они используются при изучении спроса на различные виды товаров легкой промышленности, а также для моделирования зависимости величины спроса от уровня расходов на рекламу и других факторов. Необходимость применения эконометрических методов стала актуальной при изучении состояния и перспектив развития рынка промышленной игрушки.

Специфика отрасли, особенности ее развития отражаются на возможности и целесообразности применения конкретных методов, моделей, информационных технологий, способов обработки статистической информации, анализа и прогнозирования ее индикаторов.

\section{Исторические этапы развития производства промышленной игрушки}

Для более полной характеристики отрасли необходимо вспомнить исторические этапы развития производства промышленной игрушки.

К началу 1990-х гг. промышленное производство отечественной игрушки стало резко сокращаться. Потребительский рынок начал насыщаться за счет ускорившегося притока в страну импортных изделий. Произошло временное вытеснение с рынка отечественных товаропроизводителей. К середине 1990-х гг. Значительная часть государственных предприятий перешла в частные руки, в частности, большинство предприятий, ранее выпускавших игрушки, стали коммерческими. Финансирова- ние этих предприятий из госбюджета прекратилось, вследствие чего началось резкое сокращение рабочих мест, что привело к снижению выпуска в целом отечественной игрушечной продукции. Рынок игрушек пополнялся за счет изделий, сделанных в Китае, Kopeе, Гонконге и т. д. Магазины были заполнены игрушками зарубежных товаропроизводителей.

В конце 1990-х гг. ситуация начала меняться. Согласно оценкам экспертов, позитивным последствием кризиса 1998 г. стало повышение конкурентоспособности российской экономики. Вследствие девальвации рубля цены на импортные товары внутри страны выросли, а цены отечественных товаров за границей упали, что позволило отечественным товаропроизводителям занять рынки, которые они не могли занять раньше.

В частности, рынок детских товаров и его существенная часть - рынок игрушки оказался в безраздельном пользовании российских производителей. Игрушка отечественного производителя начала пользоваться ажиотажным спросом (высокое качество, безопасность и т. д.). Для покрытия запросов торговли предприятиям пришлось пойти на значительное увеличение объемов выпуска. Лишившись импортной продукции, которая успешно продавалась на протяжении нескольких лет, торговля повысила требования к аналогичной продукции российских производителей: стала требовать ярких, броских, красочных игрушек в эффектной упаковке. Возникла необходимость обновления модельного ряда выпускаемых игрушек, причем не последнюю роль в этом стал играть не столько сам факт обновления ассортимента, сколько его динамика. Немно- 
гие предприятия смогли оборудовать высокотехнологичные пресс-формы [2].

До 90-х гг. XX в. разработкой новых образцов игрушек занимались художники Всесоюзного научно-исследовательского института игрушки. В институте разрабатывали новые технологии, изготавливали оснастку. С закрытием института многим отечественным предприятиям пришлось самостоятельно решать проблему создания и выпуска новых видов игрушки.

Для промышленного производства игрушек требуются точность, высокие технологии, использование новых материалов, изменение способов их обработки, внедрение новых технических идей и решений. Работа только над формами в условиях сокращения штата профессиональных мастеров затягивается от 4-5 месяцев до года. В то же время у зарубежных производителей игрушек весь цикл занимает 3-4 месяца (от принятия решения о выпуске нового изделия до его появления на складе).

Под эти требования наиболее подошли игрушки, произведенные на предприятиях новой волны отечественной индустрии. Частные фирмы, изначально создававшиеся для выпуска конкурентоспособной продукции, оказались в более выигрышном положении, чем бывшие государственные предприятия, обремененные издержками прошлой системы. В связи с недостаточностью инвестиционных вложений в инновационные разработки отечественные предприятия начали производить более простые изделия: пластмассовые конструкторы, ведра, лопатки, деревянные кубики, которые не требуют больших инвестиций.

Российская игрушка в переходный период времени представляла интерес для рынка только потому, что была существенно дешевле импортной. В ассортименте преобладали изделия, запущенные в производство еще в 1970-1980-е гг. На упрощение ассортимента приходилось идти по экономическим причинам.
Рынок игрушки начал активно развиваться, но скорость обновления ассортимента оказывалась недостаточной.

Одна из проблем отечественной игрушки - отсутствие известных товарных марок (брендов). Зарубежные производители детской продукции на рынок выводят те товары, которые имеют марку, пользующуюся известностью на мировом рынке.

Начиная с 2012 г. наметилась тенденция к улучшению ситуации на рынке промышленной игрушки. Отечественных производителей стали приглашать на международные выставки. Продукция отечественных игрушечных фирм стала вполне конкурентоспособной на западном рынке. Сейчас российская игрушка экспортируется в 50 стран мира.

Производством игрушек в настоящее время занимаются как предприятия, созданные еще в доперестроечные времена, а также новые фирмы, созданные во время экономических реформ. Всего в Российской Федерации около 100 крупных, средних и малых предприятий - производителей игрушек. Лидерами-производителями стали АО «Весна», АО «Звезда», завод игрушек «Огонек» и др.

Среди российских фабрик игрушек немало предприятий, которые специализируются на народных промыслах: Богородская фабрика, предприятие «Климо», «Царицынская игрушка» и др.

Для того чтобы занять устойчивое положение на мировом рынке игрушек, российским производителям необходимо работать над качеством продукции, сокращать цикл разработки новых игрушек и внедрения их на рынок, создавать собственные торговые марки.

Подводя итоги вышесказанному, отметим, что российские предприятия, производящие игрушки, претерпели много трудностей, и сейчас необходимо оперативно и грамотно оценивать сложившуюся ситуацию для возможности корректировки их деятельности с целью формирования устойчивых позиций на рынке в будущем. 
Возможность применения

и целесообразность использования эконометрических методов в анализе рынка современной промышленной

\section{игрушки}

Решение данных задач невозможно без проведения глубокого, научно обоснованного анализа состояния рынка промышленной игрушки и оценки перспектив его развития. Для этого целесообразно использование эконометрических методов, т. е. методов исследования взаимосвязей между различными экономическими процессами и явлениями с целью выявления наиболее существенных факторов, влияющих на различные экономические процессы, а также для прогнозирования показателей развития отрасли.

Далеко не все фирмы, занимающиеся производством игрушки, могут позволить себе (отсутствие высококвалифицированных специалистов-аналитиков и т. д.) проводить полноценные эконометрические исследования, охватывающие все стороны финансово-хозяйственной деятельности предприятия, производящего промышленную игрушку. Подобный анализ, как правило, проводится наиболее крупными фирмами, имеющими в штате сотрудников, обладающих знаниями в области статистического анализа и эконометрики.

Эконометрические исследования отдельных фирм в основном ориентированы на анализ информации о деятельности самого предприятия на рынке, а также на его рейтинг среди предприятий, производящих аналогичную продукцию в определенном регионе.

Так как исследуемая отрасль претерпевает множество различных трудностей (рост конкуренции, в основном с зарубежными фирмами; нехватка квалифицированных специалистов, ограниченность информационных потоков и финансов), то на широкомасштабные исследования (например, на маркетинговые исследования) не хватает ресурсов.

Руководители фирм до сих пор, основываясь лишь на своем собственном опыте, логике и умозаключениях, делают выводы о необходимости сужения или расширения ассортимента предлагаемых товаров, вывода новых видов игрушек на рынок. Однако эконометрические методы позволяют представить обоснование таких решений и снизить риск ошибочного управленческого решения.

Эконометрический анализ позволяет получить количественную оценку взаимозависимостей социально-экономических явлений и процессов. Достаточно полный обзор различных эконометрических моделей, построенных в виде уравнений множественной регрессии, описывающих подобные зависимости, представлен в работах Е. П. Голубкова [3] и Ж. Ж. Ламбена [4].

Современные пакеты прикладных программ по эконометрическому анализу дают возможность использовать основные эконометрические модели для описания текущей ситуации на рынке промышленной игрушки, динамики спроса на продукцию (промышленную игрушку), изменений структуры ассортимента.

Эконометрические методы используются при формировании цен на новые товары. Так, например, в статье Т. В. Нечаевой «Эконометрические методы в маркетинговых экспериментах» [5] приведен пример построения эконометрической модели зависимости цены на технически сложный товар от различных технико-экономических параметров, определяющих уровень качества данного изделия.

Таким образом, используя эконометрические методы, производители промышленной игрушки в анализе своей деятельности не только имеют возможность учитывать различные факторы внутренней и внешней среды, оказывающие влияние на спрос и предложение выпускаемых изделий, но и отбирать среди них наиболее значимые.

Недостатком эконометрических моделей, используемых в анализе цен на новые игрушки, является предположение о том, что цена зависит только от свойств данной игрушки, и не учитывается то, что предприятия-конкуренты устанавливают цены 
на свой товар не только на основе его качественных характеристик.

Как отмечает Т. В. Нечаева, эконометрические методы и модели применять в маркетинговых экспериментах целесообразно в случае, когда выполняются следующие условия их реализации: существует реальная причинно-следственная зависимость между изучаемой величиной (например, объемом продаж) и набором факторов, ее определяющих; имеющиеся факторы можно выразить в виде количественно измеримых или фиктивных переменных, характеризующих качественные различия между условиями эксперимента; математическая форма зависимости между изучаемой величиной и набором факторов либо заранее известна, либо ее можно предположительно оценить (например, экспертным путем); степень влияния каждого фактора на исследуемую величину сохранит свое значение и направление в будущем; существует возможность прогнозировать или контролировать будущие изменения факторных переменных [5].

Аналитических форм представления эконометрических моделей известно достаточно много (как простейших, так и более сложных). Для анализа и прогнозирования развития современной промышленной игрушки могут применяться как классические трендовые модели (линейная, гиперболическая, степенная, экспоненциальная, полулогарифмическая и т. д.), так и более сложные функции, в том числе многофакторные регрессионные модели.

Очевидно, что для построения сложных эконометрических моделей, описывающих отдельные области производства промышленной игрушки, необходим достаточно полный ряд статистических данных. Но в то же время далеко не всегда можно найти полную и достоверную информацию, которая необходима предприятиям для оценки возможности успешного развития на конкретном рынке. Зачастую необходим сбор дополнительной информации, например, путем проведения маркетинговых экспериментов, пробных продаж, па- нельных исследований доходов и расходов потребителей и т. д.

Эконометрическая модель любого макро- или микроэкономического процесса может быть представлена в виде системы эконометрических уравнений, под которой, как правило, понимается система одновременных, совместных уравнений. Ее применение на практике в анализе состояния и развития современной промышленной игрушки имеет сложности, которые сопряжены с ошибками спецификации модели (ввиду большого числа факторов).

Формируя модель конкретного экономического объекта в виде набора экзогенных и эндогенных переменных, исследователь описывает ситуацию, которая сложилась в данный момент, но она может измениться. Поэтому может измениться и вид эконометрической модели с точки зрения ее идентифицируемости.

Сверхидентифицируемые модели, используемые в анализе развития современной промышленной игрушки, можно привести к виду точно идентифицируемых за счет добавления переменных или отбрасывания отдельных ограничений на параметры. В то же время при правильной спецификации модели она может оказаться неидентифицируемой, и появится целесообразность перехода к сверхидентифицируемым или точно идентифицируемым моделям (несколько упрощающим характер взаимосвязей экономических явлений).

Необходимо принять во внимание, что для решения одного и того же класса задач имеется множество моделей. Наиболее ярко это проявляется при построении макроэкономических моделей, которые также можно применять для анализа развития современной промышленной игрушки (например, когда требуется построить уравнение регрессии, выражающее зависимость расходов населения на непродовольственные товары от различных факторов).

Далеко не все эконометрические модели имеют вид системы одновременных уравнений. Например, широкий класс функ- 
ций спроса на ряд потребительских товаров часто представляет собой рекурсивную систему, в которой с уравнениями можно работать последовательно, поэтому проблемы одновременного оценивания не возникают.

Применение тех или иных эконометрических моделей зависит от возможности получения информации, от состояния и динамики внешней и внутренней среды, в которой осуществляют свою деятельность промышленные предприятия, производимые игрушки. Для того чтобы руководители имели возможность менять сложившуюся на предприятии ситуацию в сторону улучшения, следует ориентироваться на возможности и потребности потенциального потребителя, анализ которых также возможен на основе применения эконометрических моделей [6].

\section{Применение эконометрической модели}

Рассмотрим применение эконометрической модели для анализа расходов на покупку промышленной игрушки 40 семьями города Сергиев Посад в октябре 2018 г.

В качестве результативного признака были рассмотрены расходы на покупку товаров для детей (промышленной игрушки) Y, на изменение которых влияли следующие факторы:

- $X_{1}$ - число членов семьи, чел.;

- $X_{2}$ - доход семьи, тыс. руб.;

- $X_{3}$ - число детей в семье, чел.;

- $X_{4}$ - среднедушевой доход, тыс. руб.;

- $X_{5}$ - затраты на питание, тыс. руб.

Для оценки степени тесноты и направления связи между признаками, характеризующими расходы на покупку промышленной игрушки для детей, была построена матрица парных коэффициентов корреляции (таблица).

\section{Матрица парных коэффициентов корреляции}

\begin{tabular}{|c|c|c|c|c|c|c|}
\hline & $\boldsymbol{X}_{\mathbf{1}}$ & $\boldsymbol{X}_{\mathbf{2}}$ & $\boldsymbol{X}_{\mathbf{3}}$ & $\boldsymbol{X}_{\mathbf{4}}$ & $\boldsymbol{X}_{\mathbf{5}}$ & $\boldsymbol{\gamma}$ \\
\hline $\boldsymbol{X}_{\mathbf{1}}$ & 1,000 & 0,566 & 0,836 & $-0,430$ & 0,909 & $-0,150$ \\
\hline $\boldsymbol{X}_{\mathbf{2}}$ & 0,566 & 1,000 & 0,136 & 0,468 & 0,800 & 0,661 \\
\hline $\boldsymbol{X}_{\mathbf{3}}$ & 0,836 & 0,136 & 1,000 & $-0,686$ & 0,590 & $-0,512$ \\
\hline $\boldsymbol{X}_{\mathbf{4}}$ & $-0,430$ & 0,468 & $-0,686$ & 1,000 & $-0,138$ & 0,883 \\
\hline $\boldsymbol{X}_{\mathbf{5}}$ & 0,909 & 0,800 & 0,590 & $-0,138$ & 1,000 & 0,102 \\
\hline $\boldsymbol{Y}$ & $-0,150$ & 0,661 & $-0,512$ & 0,883 & 0,102 & 1,000 \\
\hline
\end{tabular}

Анализируя значения парных коэффициентов корреляции между факторными признаками, влияющими на величину расходов семей города на покупку промышленной игрушки для детей, можно отметить, что наблюдается сильная корреляционная зависимость между числом членов семьи $\left(X_{1}\right)$ и числом детей в семье $\left(X_{3}\right)$, что совершенно логично даже на уровне логического анализа. Реализация корреляционного метода анализа только подтвердила данное предположение $\left(r_{x y}=0,836\right)$. При этом наблюдается проблема мультиколлинеарности, т. е. наличия сильной корреляционной зависимости между данными рассматриваемыми признаками, которая может быть устранена на основе анализа парных коэффициентов корреляции каждого факторного признака из пары сильно коррелированных с результативным. Данные таблицы свидетельствуют, что наиболее сильное влияние на изменение расходов на покупку промышленных товаров для детей оказывает число детей в семье $\left(X_{3}\right)$. Число членов семьи $\left(X_{1}\right)$ из дальнейшего рассмотрения будет исключено.

Сильная корреляционная зависимость также наблюдается между числом членов семьи $\left(X_{1}\right)$ и величиной затрат на питание $\left(X_{5}\right)$. В связи с тем что на предыдущем шаге исследования число членов семьи было исключено из дальнейшего рассмотрения, 
затраты на питание на этом этапе остаются для дальнейшего анализа.

Апеллируя логикой экономического анализа, нецелесообразно одновременно рассматривать для включения в одну и ту же модель доход семьи $\left(X_{2}\right)$ и среднедушевой доход $\left(X_{4}\right)$, так как один из показателей является производным от другого и тем самым информация дублируется. Исходя из этого фактор среднедушевого дохода на одного члена семьи не рассматривался.

Анализируя взаимосвязь между расходами на покупку промышленной игрушки для детей и оставшимися факторными признаками, можно отметить практически отсутствующую связь данного признака с фактором затрат на питание в семье $\left(X_{5}\right.$ $\left.r_{x y}=0,102\right)$, которая является статистически незначимой по $t$-критерию Стьюдента на уровне значимости 0,05 и тем самым исключает возможность рассмотрения данного факторного признака в дальнейшем анализе.

Таким образом, моделируя величину расходов на покупку промышленной игрушки для детей, рассмотрены и учтены следующие факторные признаки:

- $X_{2}$ - доход семьи, тыс. руб.;

- $X_{3}$ - число детей в семье, чел.

Их связь с моделируемым показателем статистически подтверждена на основе t-критерия Стьюдента на уровне значимости 0,05.

Модель регрессии величины расходов на покупку промышленной игрушки для детей семьями города Сергиев Посад имеет следующий вид:

$$
\bar{Y}_{x}=-0,031+0,295 X_{2}-1,077 X_{3} .
$$

Оценка статистической значимости построенного уравнения регрессии была осуществлена на основе F-критерия Фишера - Снедекора, в ходе реализации которого расчетное значение критерия $(14,52)$ превысило табличное $(4,46)$ на уровне значимости 0,05, что свидетельствует о статистической значимости полученной модели.

Данный вывод также был подтвержден на основе расчета и анализа средней ошибки аппроксимации, значение которой для построенной модели регрессии составило 7,7\%, что свидетельствует об ее адекватности:

$$
\bar{Y}_{x}=-0,031+0,295 X_{2}-1,077 X_{3} .
$$

Таким образом, рассмотренный пример показывает, что эконометрические методы целесообразно применять для анализа как текущей ситуации на рынке детских игрушек, так и конъюнктуры рынка, что может быть положено в основу разработки новых моделей и модификаций игрушек, определения перспективных направлений расширения и обновления ассортимента.

\section{Список литературы}

1. Базовые проблемы развития легкой промышленности (по резолюции 1-го Международного форума). - URL: http://expert.ru/northwest/2014/45/bazovyie-problemyi-razvitiyalegkoj-promyishlennosti-po-rezolyutsii-1-go-mezhdunarodnogo-foruma-legpromforum-2014

2. Гавъа Р. В., Ложкомоева Е. Н., НечаеВа Т. В. Конкурентоспособность отечественной игрушки и пути ее повышения // Вестник Университета. Государственный университет управления. - 2018. - № 1. - С. 114-116.

3. Голубков Е. П. Маркетинговые исследования: теория, методология, практика. - М. : Финпресс, 2002.

4. Ламбен Ж. Ж. Стратегический маркетинг. Европейская перспектива. - СПб. : Наука, 1997.

5. Нечаева Т. В. Эконометрические методы в маркетинговых экспериментах // Вестник МГУПИ. Сер. «Социально-экономические науки». - 2012. - № 42. - С. 119-127.

6. Ставер Н. И., Бартуш А. А. Стратегическое управление компанией в условиях «цифровой экономики» // Международный студенческий научный вестник. - 2018. № 4-6. - URL: http:/ / eduherald.ru/ru/article/view?id=19017 (дата обращения: 06.12.2018). 


\section{References}

1. Bazovye problemy razvitiya legkoy promyshlennosti (po rezolyutsii 1-go Mezhdunarodnogo foruma) [Basic Problems of Light Industry Development (according to the Resolution of the 1st International Forum]. (In Russ.). Available at: http://expert.ru/northwest/2014/45/ bazovyie-problemyi-razvitiya-legkoj-promyishlennostipo-rezolyutsii-1-go-mezhdunarodnogo-foruma-legpromforum-2014

2. Gavva R. V., Lozhkomoeva E. N., Nechaeva T. V. Konkurentosposobnost' otechestvennoy igrushki i puti ee povysheniya [Competitiveness of Domestic Toys and Ways to Improve it]. Vestnik Universiteta. Gosudarstvennyy universitet upravleniya [Bulletin of the University. State University of management], 2018, No. 1, pp. 114-116. (In Russ.).

3. Golubkov E. P. Marketingovye issledovaniya: teoriya, metodologiya, praktika [Marketing Research: Theory, Methodology, Practice]. Moscow, Finpress, 2002. (In Russ.).

4. Lamben Zh. Zh. Strategicheskiy Marketing. Evropeyskaya perspektiva [Strategic Marketing. A European Perspective]. Saint Petersburg, Nauka, 1997. (In Russ.).

5. Nechaeva T. V. Ekonometricheskie metody $\mathrm{V}$ marketingovykh eksperimentakh [Econometric Methods in Marketing Experiments]. Vestnik MGUPI. Ser. "Sotsial'noekonomicheskie nauki» [Vestnik MGUPI, Ser. "Social and Economic Sciences"], 2012, No. 42, pp. 119-127. (In Russ.).

6. Staver N. I., Bartush A. A. Strategicheskoe upravlenie kompaniey v usloviyakh «tsifrovoy ekonomiki» [Strategic Management of the Company in a "Digital Economy"]. Mezhdunarodnyy studencheskiy nauchnyy vestnik [The International Student Scientific Bulletin], 2018, No. 4-6. (In Russ.). Available at: http:/ / eduherald.ru/ru/article/view?id=19017 (accessed 06.12.2018).

\section{Сведения об авторах}

Руслан Витальевич Гавва

кандидат экономических наук, директор Сергиево-Посадского института игрушки филиала Высшей школы народных искусств (академии).

Адрес: Сергиево-Посадский институт игрушки - филиал ФГБОУ ВО «Высшая школа народных искусств (академия)», 141300, Московская область, Сергиев Посад, Северный проезд, д. 5. E-mail: gavva@rambler.ru

\section{Евгения Николаевна Ложкомоева}

кандидат экономических наук, доцент, и. о. заведующего кафедрой экономикоправовых и управленческих дисциплин Сергиево-Посадского филиала Московского педагогического государственного университета.

Адрес: Сергиево-Посадский филиал ФГБОУ ВО «Московский педагогический государственный университет», 141315, Московская область, Сергиев Посад, ул. Разина, д. 1-А. E-mail: logkomoeva_en@mail.ru

\section{Information about the authors}

\section{Ruslan V. Gavva}

$\mathrm{PhD}$, Director

of the Sergiev Posad Institute of Toys -

branch of Higher School of Folk Arts (Academy). Address: Sergiev Posad Institute of Toys branch of Higher School of Folk Arts (Academy), 5 Severnyy proezd, Sergiev Posad,

Moscow region, 141300,

Russian Federation.

E-mail: gavva@rambler.ru

\section{Evgenia N. Lozhkomoeva}

PhD, Assistant Professor, Acting Head of the Department for Economic- Legal and Managerial Studies of the Sergiev Posad branch of the Moscow Pedagogical State University.

Address: Sergiev Posad branch of the Moscow Pedagogical State University,

1-A Razina Str., Sergiev Posad,

Moscow region, 141315,

Russian Federation.

E-mail: logkomoeva_en@mail.ru 